\title{
Serviços Ambientais Gerados por Matas Tuteladas ao Exército Brasileiro na Região Metropolitana no Recife
}

\author{
Helder de Barros Guimarães ${ }^{1}$, Ricardo Augusto Pessoa Braga ${ }^{1}$, \\ Tiago Henrique de Oliveira ${ }^{2}$ \\ ${ }^{1}$ Departamento de Engenharia Civil, Universidade Federal de Pernambuco - UFPE \\ ${ }^{2}$ Departamento de Ciências Geográficas, Universidade Federal de Pernambuco - UFPE
}

\section{RESUMO}

Este trabalho tem o objetivo de analisar a evolução dos serviços ambientais climáticos gerados por quatro fragmentos de Mata Atlântica tutelados ao Exército Brasileiro localizados na Região Metropolitana do Recife, por meio da comparação dos valores da Temperatura da Superfície (Ts) gerados a partir de imagens do satélite TM Landsat 5, em intervalos de tempo que variam de 19 a 21 anos. Antes de ficarem sob a responsabilidade do Exército, as áreas de estudo eram utilizadas para o cultivo de cana-de-açúcar e capim para pecuária. Conclui-se que as condições climáticas no interior dos fragmentos analisados melhoraram com o decorrer dos anos e que o método de comparação da Temperatura Superficial por análise do balanço de radiação mostrou-se adequado ao estudo comparativo da evolução ambiental de fragmentos de mata.

Palavras-chave: serviços ambientais, mata atlântica, fragmento florestal, exército brasileiro, geoprocessamento.

\section{Environmental Services Generated by Forests Protected by the Brazilian Army Located in the Metropolitan Area of Recife}

\begin{abstract}
The purpose of this research was to analyze the evolution of the climatic environmental services generated by four fragments of the Atlantic rainforest under the protection of the Brazilian Army, located in the metropolitan area of Recife, through the comparison of Surface Temperatures (St) and Enhanced Vegetation Index (EVI) generated as from the images captured by the TM Landsat 5 satellite in time periods ranging from 19 to 21 years. Before being under the responsibility of the Army, the areas studied were used for the growth of sugarcane and grassland for livestock. It was possible to conclude that the climatic conditions within the fragments analyzed have improved over the years, and that the Surface Temperature comparison method through the analysis of the radiation balance was considered appropriate to the comparative study of the environmental evolution of forest fragments.
\end{abstract}

Keywords: environmental services, atlantic rainforest, forest fragment, Brazilian Army, geoprocessing (GIS). 


\section{INTRODUÇÃO}

A expressão Mata Atlântica foi proposta pela primeira vez em 1884 por J. E. Wappaeus para definir a mata costeira perenifólia higrófila, podendo esta estar situada sobre serras ou não, por vezes em baixas altitudes e até um pouco acima do nível do mar (Urbanetz, 2005). Estima-se que, no início do século XVI, o bioma Mata Atlântica cobria cerca de $15 \%$ da superfície atual do território brasileiro, estendendo-se do Estado do Maranhão até a região Sudeste do Brasil. No início da colonização brasileira, no século XVI, a matriz econômica da época estava alicerçada na extração do pau-brasil, uma espécie endêmica desse bioma, que possuía grande valor e era utilizada como corante e na construção civil. $\mathrm{O}$ processo de colonização concentrou-se na região costeira do Brasil, justamente no local onde a Mata Atlântica ocorria na sua origem. Com a colonização impulsionada pelos ciclos do café, da cana-de-açúcar e do algodão, nos séculos XVII e XVIII, e posteriormente pelo intenso processo de urbanização e expansão agrícola dos séculos XIX e XX, a Mata Atlântica foi devastada, reduzindo-se a apenas menos de $8 \%$ de sua área original (Lino, 2003)

Os processos de desflorestamento sofridos pela Mata Atlântica nos últimos séculos fizeram com que esse bioma se resumisse a fragmentos florestais dispersos no seu espaço original. Tal fragmentação introduziu uma série de novos fatores na história evolutiva de populações naturais de plantas e animais. Essas mudanças afetaram de forma diferenciada os parâmetros demográficos de mortalidade e natalidade de diferentes espécies e, portanto, a estrutura e a dinâmica de ecossistemas (Viana \& Pinheiro, 1988).

Os Serviços Ambientais são definidos como interações e processos naturais que satisfazem e sustentam a vida humana (De Groot, 1992). Esses serviços podem ser gerados basicamente por três fontes: as florestas, o oceano e as terras agrícolas ou cultivadas.

Para Cavalcanti (2002), existem basicamente três tipos de serviços ambientais: a manutenção da biodiversidade, a manutenção dos estoques de carbono e o ciclo da água. Os mesmos, segundo Tonhasca (2004), podem ser exemplificados pelo controle de erosão e sedimentação por meio da retenção do solo, pela regulação do fluxo hidrológico, pelo controle de distúrbios climáticos, pelo valor cultural e estético, pela proteção de habitat, pelo controle de doenças e pragas, e pela fonte de material genético.

O sensoriamento remoto tem ajudado no monitoramento espaçotemporal dos recursos naturais em diversas partes do mundo de maneira eficiente e sistemática (Baldocchi \& Meyers, 1998; Accioly et al., 2002; Giongo, 2008; Oliveira et al., 2010), vindo a apresentar grande auxílio também nas atividades humanas, como a agropecuária (Leitão \& Oliveira, 2000; Almeida et al., 2005) e a análise de áreas urbanas (Camargo et al., 2007).

Desse modo, diversos índices têm sido apresentados mais recorrentemente na literatura visando à avaliação dos recursos naturais, como os índices de vegetação, a medição do albedo superficial e a estimativa da temperatura da superfície (Oke, 1987; Asner, 2001; Paiva, 2005; Silva et al., 2005a, b; Oliveira et al., 2010). Merece destaque o Enhanced Vegetation Index (EVI), pois é um índice discriminador de vegetação caracterizado por ser mais sensível à variação da estrutura do dossel, além de promover uma redução das influências atmosféricas e do fundo do dossel no monitoramento da vegetação (Justice et al. 1998).

Nesse contexto, este trabalho tem o objetivo de analisar a evolução dos serviços ambientais climáticos gerados por quatro fragmentos de Mata Atlântica tutelados ao Exército Brasileiro localizados na Região Metropolitana do Recife, por meio da comparação dos valores da Temperatura da Superfície (Ts) gerados a partir de imagens do satélite TM Landsat 5 , em períodos que variam de 19 a 21 anos.

\section{MATERIAIS E MÉTODOS}

\section{1. Áreas de estudo}

Foram alvo desta pesquisa quatro fragmentos de Mata Atlântica, que são utilizados pelo Exército Brasileiro para a realização de manobras militares. Um dos fragmentos está localizado em uma região rural e os demais estão inseridos na parte urbana da 
Região Metropolitana do Recife-PE. As áreas estão sob a responsabilidade das seguintes Organizações Militares (OM): Campo de Instrução 'Marechal Newton Cavalcanti' (CIMNC), 4 Batalhão de Comunicações ( $\left.4^{\circ} \mathrm{BCom}\right), 14^{\circ}$ Batalhão de Infantaria Motorizado (14 BIMtz) e o Complexo Militar do Curado [constituído pelo Comando Militar do Nordeste (CMNE), pelo $4^{\circ}$ Batalhão de Polícia do Exército ( $4^{\circ} \mathrm{BPE}$ ) e pelo $10^{\circ}$ Esquadrão de Cavalaria Mecanizado (10 Esqd C Mec)].

A Tabela 1 apresenta um resumo de dados relativos às áreas, aos aspectos, à antiga destinação de uso do solo, à localização e quando essas áreas passaram à tutela do Exército.

\subsection{Metodologia}

Com o objetivo de gerar mapas temáticos que possibilitassem identificar a evolução da condição ambiental das áreas de estudo, foram utilizadas imagens TM do satélite Landsat 5 referentes ao período de 1988 a 2010, adquiridas junto ao Instituto Nacional de Pesquisas Espaciais (INPE), de órbita e ponto 214/65-66. As passagens do satélite sobre as áreas de estudo ocorreram em 5 de junho de 1988, 28 de setembro de 1989, 26 de agosto de 2006, 07 de abril de 2007 e 06 de setembro de 2010.

As imagens do sensor TM do satélite Landsat-5 constam de sete bandas espectrais, das quais se utilizou a banda 6, que compreende a faixa do infravermelho (10,4 e 12,5 $\mu \mathrm{m})$. Essa banda apresenta sensibilidade aos fenômenos relativos aos contrastes térmicos, servindo para detectar propriedades termais de rochas, solos, vegetação, água, concreto e zinco, dentre outros.

Desse modo, foram obtidas as Temperaturas da Superfície (Ts) e os Enhanced Vegetation Index (EVI) para as áreas de estudo empregando-se a mesma metodologia utilizada por Silva et al. (2005a, b) e Oliveira \& Galvíncio (2008), demonstrada por meio do fluxograma da Figura 1, no qual foram desenvolvidos modelos com a ferramenta Model Maker do programa ERDAS Imagine 9.3.

As sete etapas desse processo são descritas a seguir:

\subsubsection{Radiância Espectral}

A radiância espectral ou a calibração radiométrica (Equação 1) consiste na conversão do nível de cinza de cada pixel e banda em radiância monocromática, que representa a energia solar refletida, por unidade de área, de tempo, de ângulo sólido e de comprimento de onda, medida no nível do satélite nas bandas 1, 2, 3, 4, 5 e 7. Para a banda termal, essa radiância representa a radiação emitida por cada pixel, sendo empregada na obtenção da temperatura da superfície. A radiância de cada pixel e banda foi obtida pela equação proposta por Markhanm \& Baker (1987):

$L_{\lambda i}=a_{i}+\frac{b_{i}-a_{i}}{255} N D$

em que: $a$ e $b=$ radiâncias espectrais mínimas e máximas $\left(\mathrm{Wm}^{-2} \mathrm{sr}^{-1} \mu \mathrm{m}^{-1}\right)$; $\mathrm{ND}=$ intensidade do pixel (número inteiro compreendido entre 0 e 255); $\mathrm{i}=$ bandas $(1,2, \ldots$ e 7$)$ do satélite Landsat 5 .

Tabela 1. Informações sobre as áreas de estudo.

Table 1. Information on areas of study.

\begin{tabular}{|c|c|c|c|c|}
\hline $\begin{array}{l}\text { Organização } \\
\text { militar }\end{array}$ & $\begin{array}{l}\text { Área } \\
\text { (ha) }\end{array}$ & $\begin{array}{l}\text { Antiga destinação } \\
\text { do uso do solo }\end{array}$ & Localização & $\begin{array}{l}\text { Quando passou à } \\
\text { tutela do exército }\end{array}$ \\
\hline CIMNC & $7.342,00$ & Engenhos de cana-de-açúcar & $\begin{array}{c}7^{\circ} 49^{\prime} 52^{\prime \prime} \mathrm{S} \\
53^{\circ} 06^{\prime} 09^{\prime \prime} \mathrm{W}\end{array}$ & 1944 \\
\hline $4^{\circ} \mathrm{BCom}$ & 173,53 & $\begin{array}{l}\text { Fazenda do Ministério da Agricultura } \\
\text { destinada ao cultivo de capim }\end{array}$ & $\begin{array}{l}8^{\circ} 05^{\prime} 59^{\prime \prime} \mathrm{S} \\
34^{\circ} 57^{\prime} 22^{\prime \prime} \mathrm{W}\end{array}$ & 1965 \\
\hline $14^{\circ}$ BIMtz & 401,40 & Conjunto de pequenos sítios & $\begin{array}{l}8^{\circ} 05^{\prime} 34^{\prime \prime} \mathrm{S} \\
35^{\circ} 00^{\prime} 18^{\prime \prime} \mathrm{W}\end{array}$ & 1958 \\
\hline $\begin{array}{l}\text { Complexo Militar do } \\
\text { Curado }\end{array}$ & 340,00 & $\begin{array}{l}\text { A propriedade pertencia ao Instituto } \\
\text { de Pesquisa e Experimentação Agro- } \\
\text { pecuária do Nordeste e destinava-se ao } \\
\text { cultivo de cana-de-açúcar e capim }\end{array}$ & $\begin{array}{l}8^{\circ} 04^{\prime} 18^{\prime \prime} \mathrm{S} \\
34^{\circ} 57^{\prime} 53^{\prime \prime} \mathrm{W}\end{array}$ & 1972 \\
\hline
\end{tabular}

Fonte: (Guimarães, 2011). 


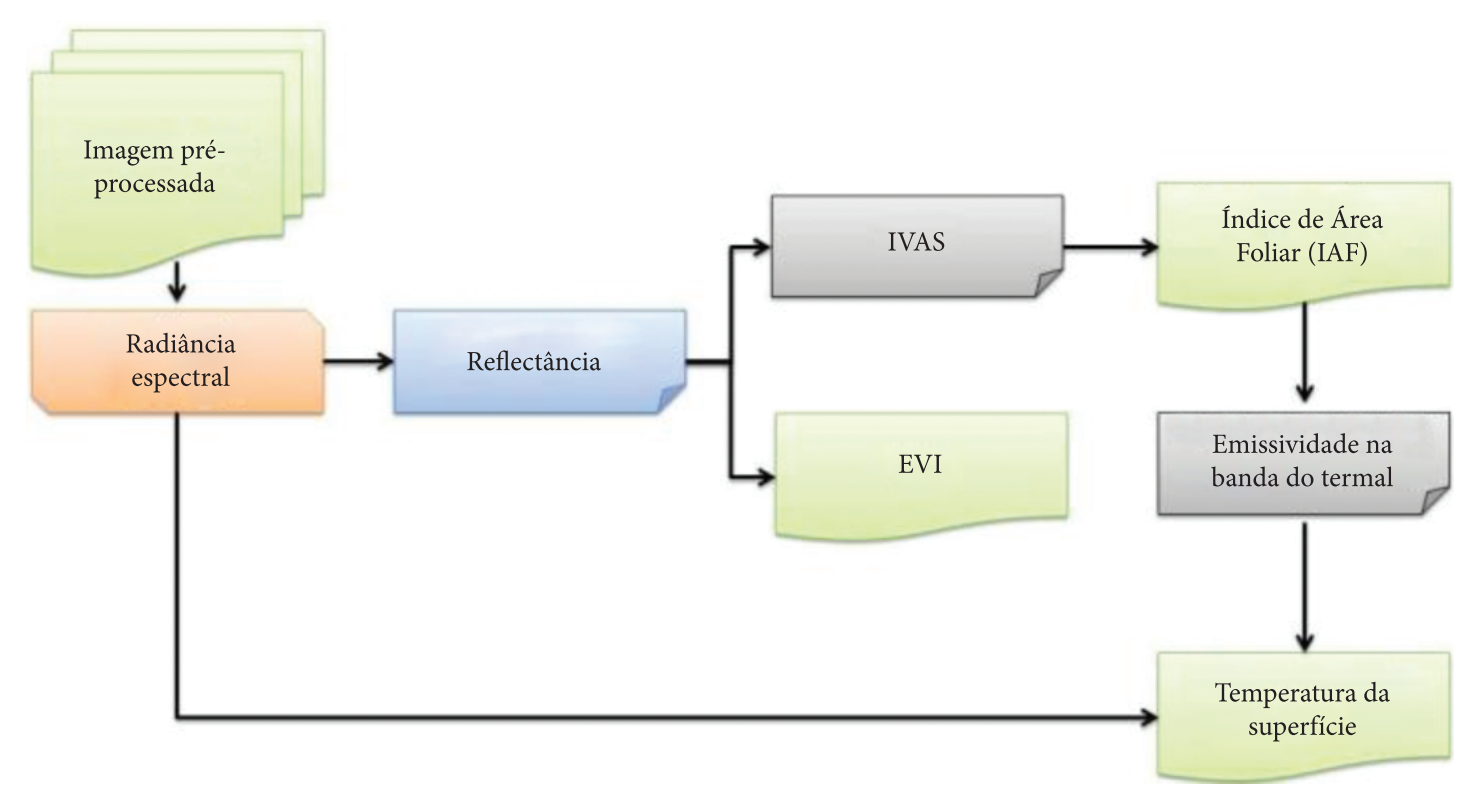

Figura 1. Fluxograma da metodologia para obtenção da Temperatura da Superfície.

Figure 1. Flowchart of the method for obtaining the Surface Temperature.

Os coeficientes de calibração utilizados para as imagens TM são os propostos por Chander \& Markham (2003).

\subsubsection{Reflectância}

A reflectância (Equação 2) de cada banda é definida como sendo a razão entre o fluxo de radiação solar refletido pela superfície e o fluxo de radiação solar global incidente, a qual é obtida por meio da equação (Allen et al., 2002):

$$
\rho_{\lambda i}=\frac{\pi \times L_{\lambda i}}{K_{\lambda i} \times \cos Z \times d_{r}}
$$

em que: $\mathrm{L} \lambda \mathrm{i}=$ radiância espectral de cada banda; $\mathrm{k}_{\lambda \mathrm{i}}=$ irradiância solar espectral de cada banda no topo da atmosfera $\left(\mathrm{Wm}^{-2} \cdot \mu \mathrm{m}^{-1}\right) ; \mathrm{Z}=$ ângulo zenital solar; $\mathrm{dr}=$ quadrado da razão entre a distância média Terra-Sol ( $r_{o}$ ) e a distância Terra-Sol (r) em dado dia do ano (DSA).

\subsubsection{SAVI}

Foi utilizado o índice de vegetação ajustado por solo (Soil Adjusted Vegetation Index - SAVI) introduzindo-se um fator no IVDN para incorporar o efeito da presença do solo, mantendo-se o valor do IVDN dentro de -1 a +1 , e seguindo a equação proposta por Huete (1988). Esse índice é calculado por meio da Equação 3:

$$
S A V I=\frac{(1+L)\left(\rho_{I V}-\rho_{V}\right)}{\left(L+\rho_{I V}+\rho V\right)}
$$

em que: $\mathrm{p}_{\mathrm{iv}}$ e $\mathrm{p}_{\mathrm{v}}$ correspondem, respectivamente, às bandas do infravermelho próximo e do vermelho; $\mathrm{L}=$ constante, cujo valor mais frequentemente usado é 0,5 (Accioly et al., 2002; Boegh et al., 2002).

\subsubsection{IAF}

O Índice de Área Foliar (IAF) é definido pela razão entre a área foliar de toda a vegetação por unidade de área utilizada por ela. O IAF é um indicador da biomassa de cada pixel da imagem e o mesmo foi computado pela Equação 4, obtida por Allen et al. (2002):

$I A F=-\frac{\left(\frac{0,69-S A V I}{0,59}\right)}{0,91}$

\subsubsection{Emissividades da Superfície}

Para a obtenção da temperatura da superfície, é utilizada a equação de Planck invertida, válida para um corpo negro. Na medida em que cada pixel não emite radiação eletromagnética como um corpo negro, há a necessidade de introduzir a emissividade de cada pixel no domínio espectral da banda termal $\varepsilon_{\mathrm{NB}}$, qual seja: $10,4-12,5 \mu \mathrm{m}$. Por sua vez, quando 
do cômputo da radiação de onda longa emitida por cada pixel, há de ser considerada a emissividade no domínio da banda larga $\varepsilon_{0}(5-100 \mu \mathrm{m})$. Segundo Allen et al. (2002) apud Silva et al. (2005b), as emissividades $\varepsilon_{\mathrm{NB}}$ e $\varepsilon_{0}$ podem ser obtidas, para $\mathrm{NDVI}>0$ e IAF $<3$, segundo as Equações 5 e 6 :

$\varepsilon_{\mathrm{NB}}=0,97+0,00331 \mathrm{IAF}$

$\varepsilon_{0}=0,95+0,01 \mathrm{IAF}$

Para pixels com IAF $\geq 3, \varepsilon_{\mathrm{NB}}=\varepsilon_{0}=0,98$. Para corpos de água (IVDN $<0$ ), no caso do lago de Sobradinho e do leito do Rio São Francisco, Silva \& Cândido (2004) utilizaram os valores de $\varepsilon_{\mathrm{NB}}=0,99 \mathrm{e}$ $\varepsilon_{0}=0,985$, conforme Allen et al. (2002).

\subsubsection{Temperatura da superfície}

Para a obtenção da temperatura da superfície (Ts ), são utilizadas a radiância espectral da banda termal $\mathrm{L}_{\lambda, 6}$ e a emissividade $\varepsilon_{\mathrm{NB}}$, obtida esta na etapa anterior. Dessa forma, obtém-se a temperatura da superfície (K) pela seguinte expressão (Equação 7):

$$
T_{s}=\frac{K_{2}}{\left(\frac{\varepsilon_{N B} K_{1}}{L_{\lambda, 6}}+1\right)}
$$

em que $\mathrm{K}_{1}=607,76 \mathrm{Wm}^{-2} \mathrm{sr}^{-1} \mu \mathrm{m}^{-1}$ e $\mathrm{K}_{2}=1260,56 \mathrm{~K}$ são constantes de calibração da banda termal do Landsat 5 - T (Allen et al., 2002).

\subsubsection{EVI}

O Enhanced Vegetation Index (EVI) pode ser obtido pela Equação 8, proposta por Huete et al. (1997):

$E V I=G\left(\frac{\rho_{\mathrm{IV}}-\rho_{\mathrm{V}}}{\rho_{\mathrm{IV}}+C 1 * \rho_{\mathrm{V}}-C 2 * \rho A+L}\right)$

em que: $\rho_{\mathrm{IV}}=$ reflectância no infravermelho próximo; $\rho_{\mathrm{v}}=$ reflectância no vermelho; $\rho A=$ reflectância no azul; $\mathrm{C} 1$ = coeficiente de correção dos efeitos atmosféricos para o vermelho (6); C2 = coeficiente de correção dos efeitos atmosféricos para o azul $(7,5) ; \mathrm{L}=$ fator de correção para a interferência do solo (1); $\mathrm{G}=$ fator de ganho $(2,5)$.

A escolha desse índice de vegetação se dá por causa de sua capacidade de minimizar o efeito background do solo e os efeitos atmosféricos em função da utilização da banda do azul. Segundo Justice et al. (1998), esse índice ainda apresenta uma alta sensibilidade a variação fenológicas.

\section{RESULTADOS}

Todas as áreas de estudo foram adquiridas ou cedidas ao Exército entre as décadas de 1930 e 1960. Algumas, como as do $4^{\circ}$ BCom e do Complexo Militar do Curado, pertenciam a instituições estatais de desenvolvimento agropecuário. Desse modo, foi identificado que, nas suas origens, as mesmas possuíam pequenas áreas utilizadas por monoculturas, como a cana-de-açúcar e o cultivo de capim para alimentação de gado. Quanto à existência de traços de vegetação de Mata Atlântica nessas áreas, os documentos analisados retratam apenas a ocorrência de pequenos fragmentos que representavam não mais que $10 \%$ das áreas originais.

Observa-se na Figura 2 que a Ts do fragmento de mata sob a responsabilidade do CIMNC apresenta temperaturas mais amenas, quando comparadas com outros alvos, como solo exposto e áreas urbanizadas. As temperaturas médias em 1988 indicam registros na faixa de $22,0{ }^{\circ} \mathrm{C}$ a $24,0{ }^{\circ} \mathrm{C}$ para o interior da área de estudo e de $25,1^{\circ} \mathrm{C}$ a $26^{\circ} \mathrm{C}$ para a área externa à mesma.

Diferentemente, no ano de 2007, as faixas de temperatura médias passam de $25,1^{\circ} \mathrm{C}$ a $26^{\circ} \mathrm{C}$ para a área interna e $>28,1^{\circ} \mathrm{C}$ para a área externa. Em consequência, constata-se que em 19 anos a variação da temperatura média intra e extramata do CIMNC passou de $1{ }^{\circ} \mathrm{C}$ para $2,5^{\circ} \mathrm{C}$.

Por meio do EVI, é possível observar que o entorno da mata apresentou, em grande parte, uma diminuição de valores do índice de vegetação. Nota-se como, na imagem de 1988, havia uma predominância de EVI > 0,60 em grande parte da imagem. Contudo, no ano de 2007, é observada uma redução nos valores de EVI, com valores variando entre 0,30 e 0,60 .

Tal quadro se deve principalmente à utilização antrópica à qual a área veio a ser submetida com o passar dos anos. Diversamente, as áreas de corpos hídricos apresentaram valores inferiores a zero, como pode ser observado também em trabalhos realizados por Machado et al. (2009) em corpos hídricos e áreas 

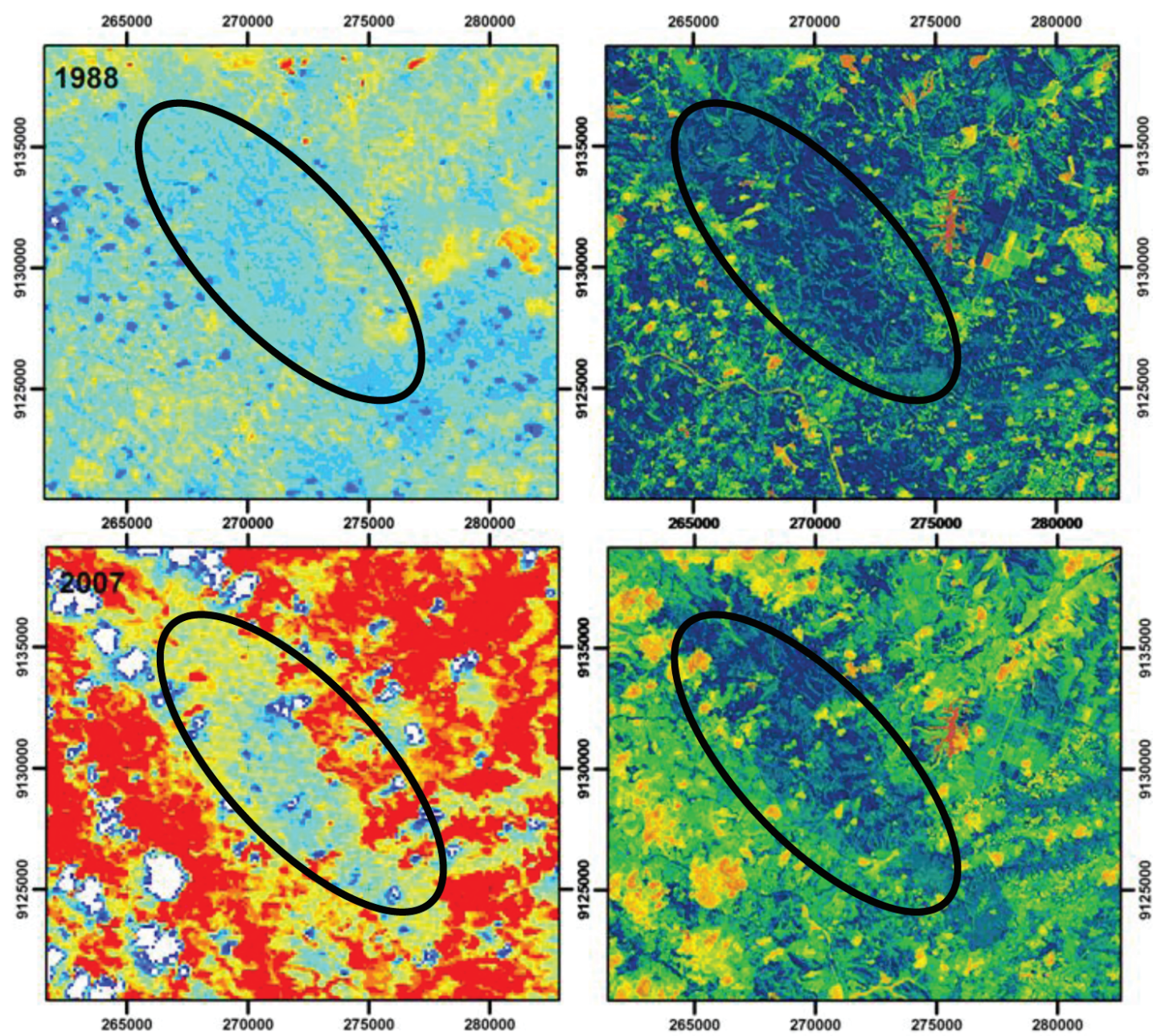

Temperatura da superfície $\left({ }^{\circ} \mathrm{C}\right)$
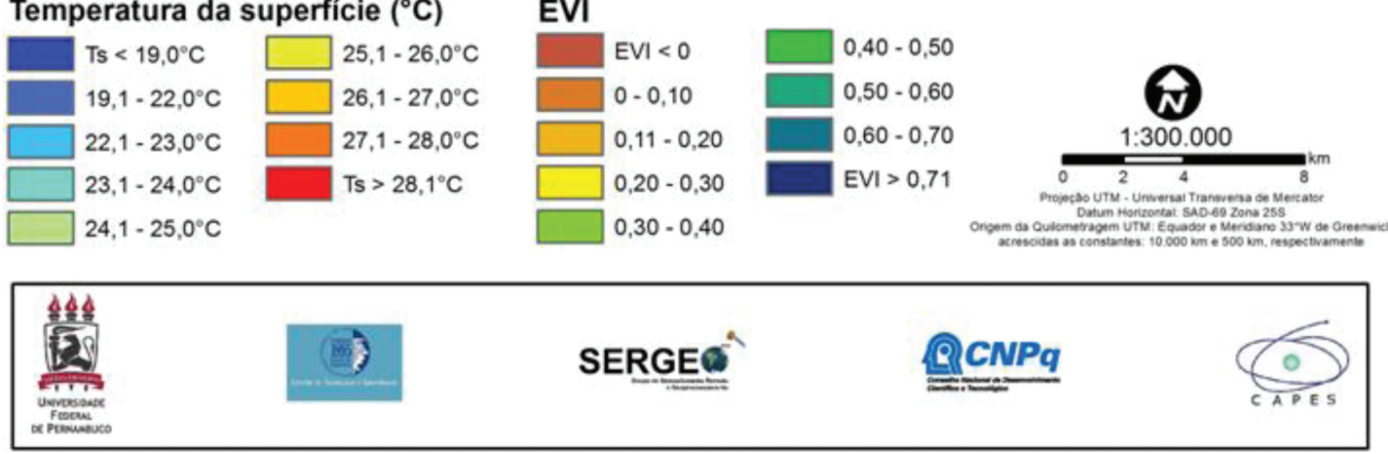

Figura 2. Variação espacial da temperatura e do EVI no CIMNC nos anos de 1988 e 2007.

Figure 2. Spatial variation of temperature and the EVI in CIMNC in the years 1988 and 2007.

com solo muito alagado em parte do município de Ladário e Corumbá-MS e por Xia et al. (2008), na planície ao norte da China.

No caso do $4^{\circ}$ BCom, totalmente inserido em uma região urbanizada, ao se compararem as Ts das imagens de 1989, 2006 e 2010 (Figura 3), pode-se observar um aumento espaçotemporal significativo da temperatura externa ao objeto de estudo, pois a mesma passa de uma faixa de $25,1^{\circ} \mathrm{C}$ a $26,0^{\circ} \mathrm{C}$ para $>28,1^{\circ} \mathrm{C}$; entretanto, observa-se uma diminuição da temperatura no interior do $4^{\circ} \mathrm{BCom}$, 

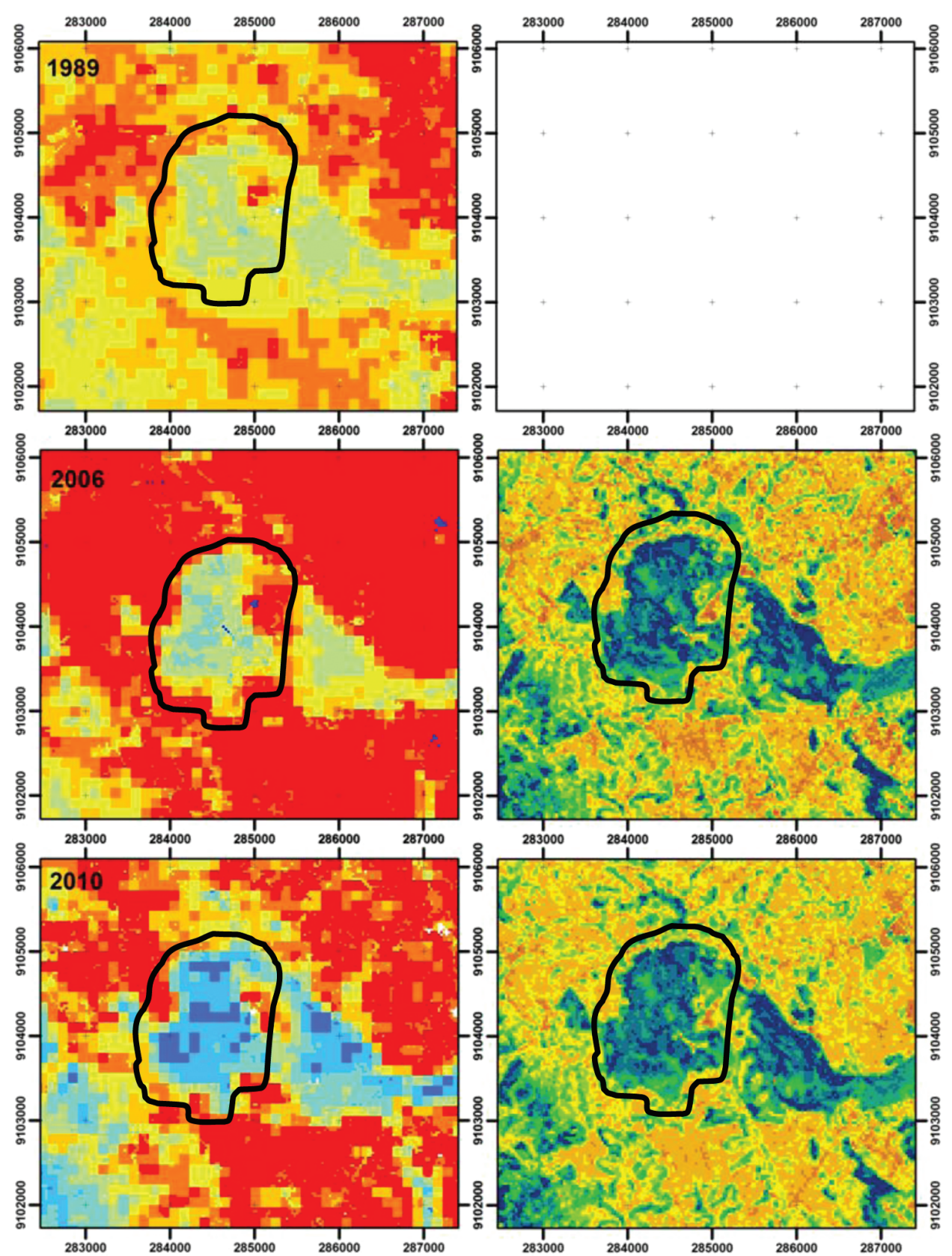

Temperatura da superficie $\left({ }^{\circ} \mathrm{C}\right)$
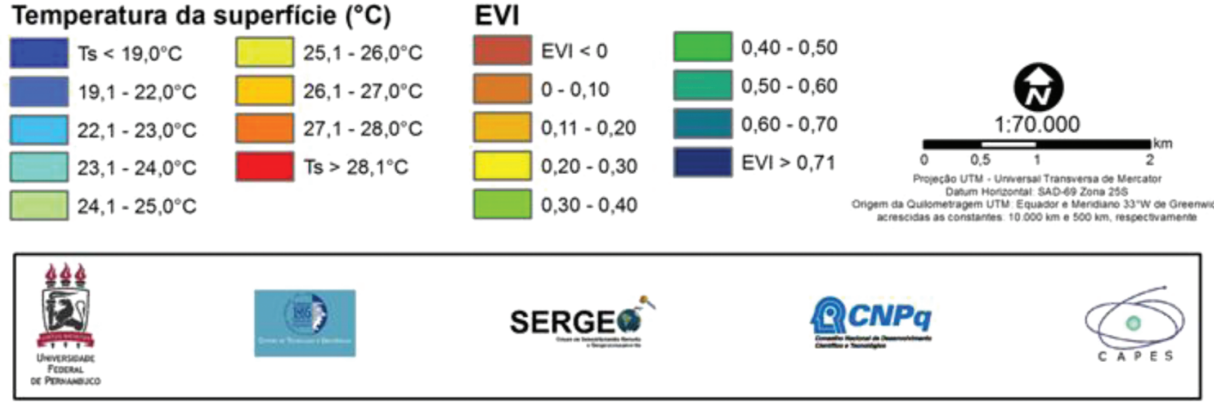

Figura 3. Variação espacial da temperatura e EVI no 4º BCom nos anos de 1989, 2006 e 2010.

Figure 3. Spatial variation of temperature and the EVI in 4º BCom in the years 1989, 2006 and 2010. 

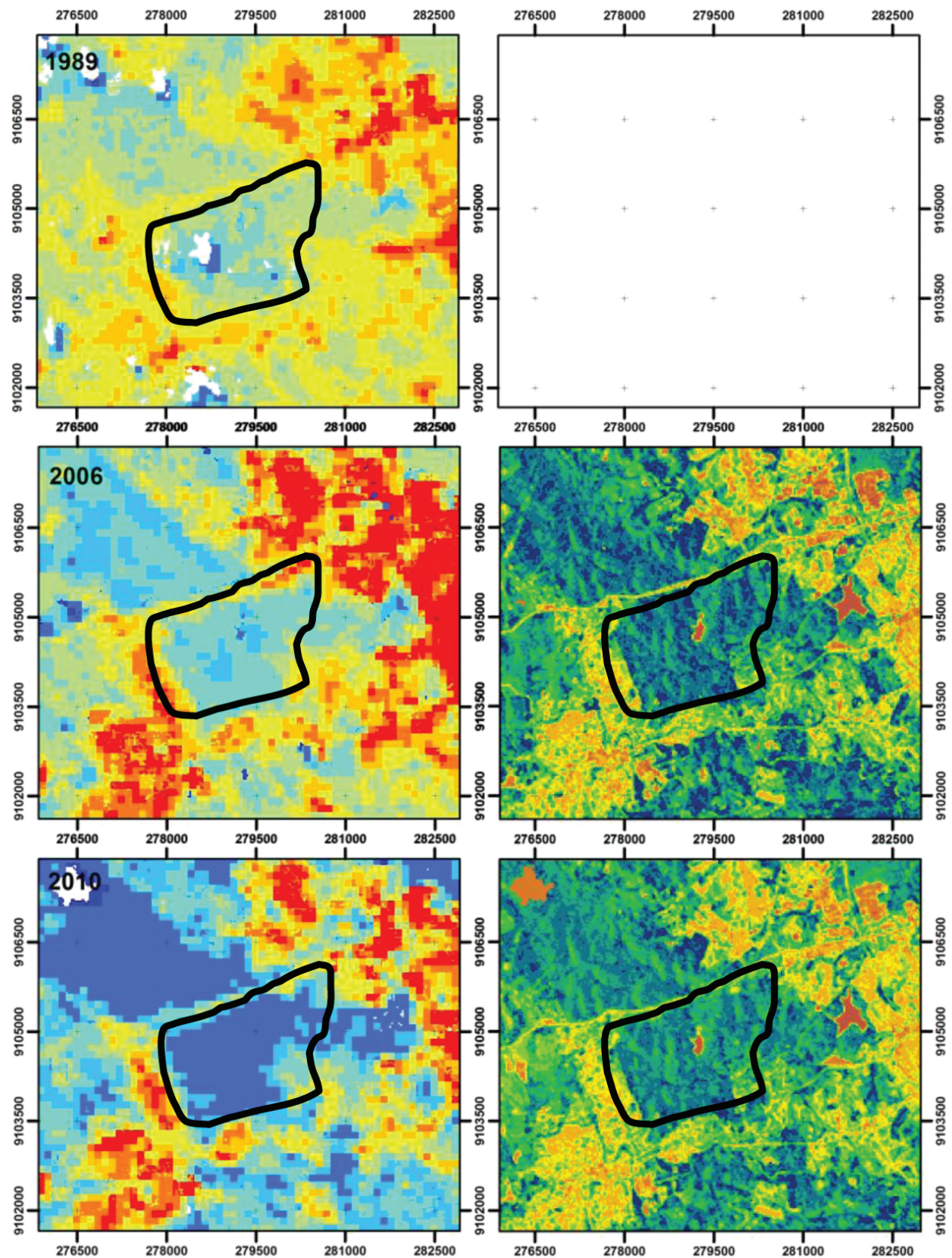

Temperatura da superfície $\left({ }^{\circ} \mathrm{C}\right)$
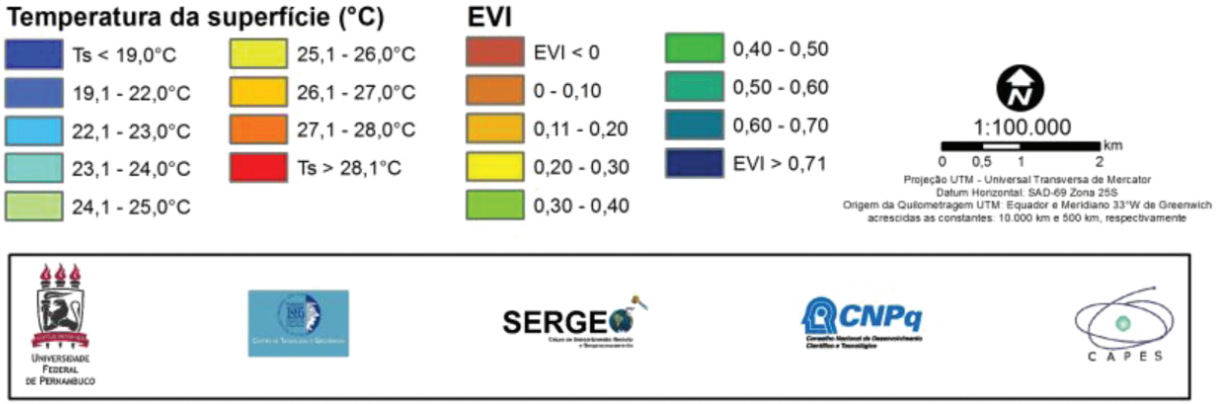

Figura 4. Variação espacial da temperatura e EVI no 14º BIMtz nos anos de 1989, 2006 e 2010.

Figure 4. Spatial variation of temperature and the EVI in 14º BIMtzin the years 1989, 2006 and 2010. 

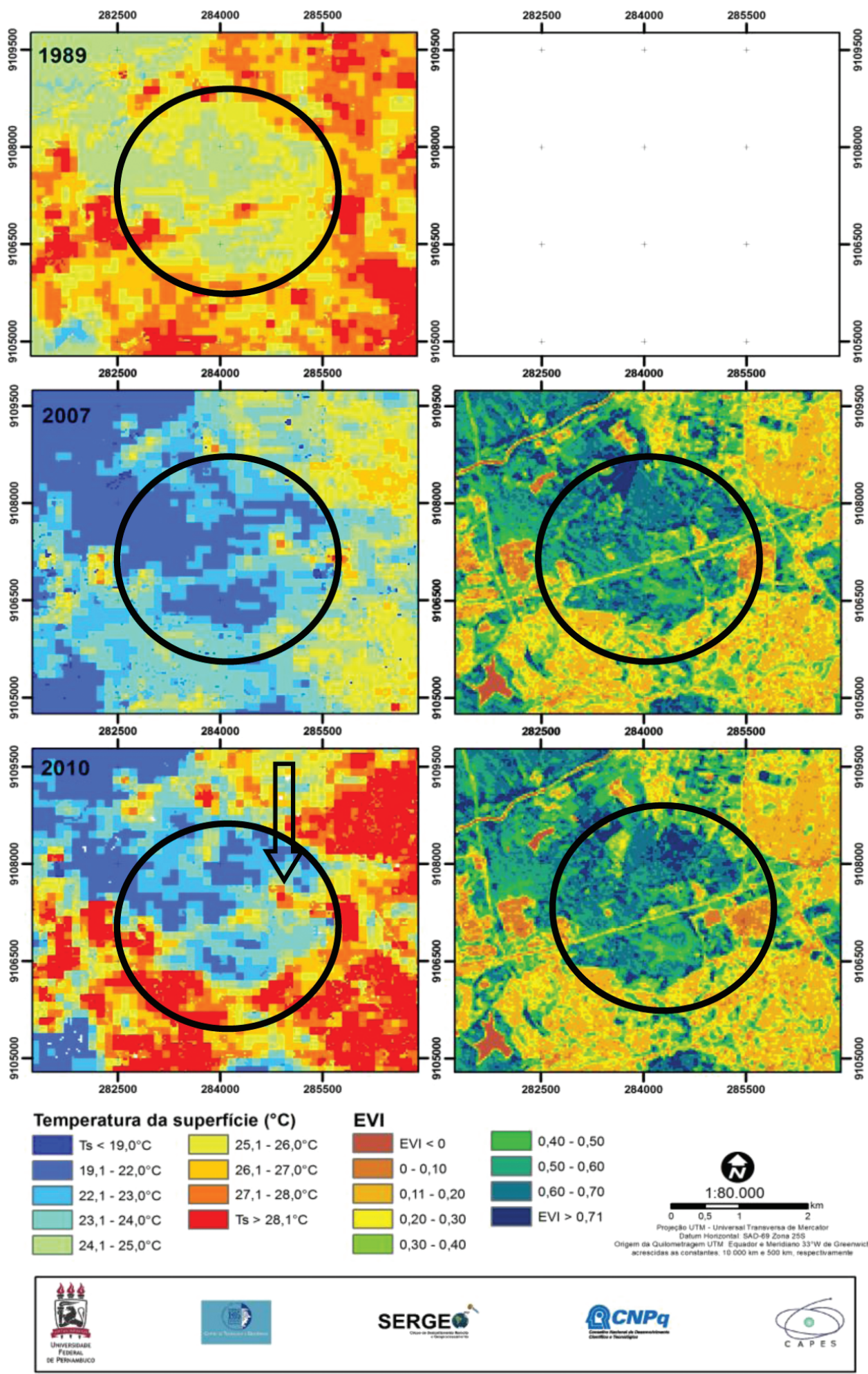

Figura 5. Variação espacial da temperatura e EVI no Complexo Militar do Curado (CMNE) nos anos de 1989, 2007 e 2010.

Figure 5. Spatial variation of temperature and the EVI in Complexo Militar do Curado (CMNE) in the years 1989, 2007 and 2010. 
pois as temperaturas médias passaram da faixa de $24,1^{\circ} \mathrm{C}$ a $25,0{ }^{\circ} \mathrm{C}$ para a faixa de $22,1^{\circ} \mathrm{C}$ a $23,0^{\circ} \mathrm{C}$.

Ou seja: para um período de aproximadamente 20 anos, houve um aumento da temperatura da área externa ao objeto de estudo na ordem de $2,5^{\circ} \mathrm{C}$ e, em contrapartida, houve uma diminuição de $2,0{ }^{\circ} \mathrm{C}$ no interior do $4^{\circ}$ BCom. Esse fato pode ser explicado por uma oportunidade de desenvolvimento de novas espécies ou um maior desenvolvimento foliar das espécies mais antigas, como pôde ser observado por Oliveira et al. (2011) na Mata da Várzea, também localizada no município do Recife-PE.

Também é observado que as áreas de solo exposto no interior do fragmento apresentaram valores de temperatura superiores a $28,1{ }^{\circ} \mathrm{C}$ e, no entorno, houve variação entre $26,1{ }^{\circ} \mathrm{C}$ e $28,0{ }^{\circ} \mathrm{C}$. É possível observar nas cartas de EVI que, no entorno dessa área de solo exposto, os valores de EVI apresentamse menos elevados $(0,30$ - 0,50) do que na área de mata densa $(E V I>0,60)$. Também são observadas no interior do fragmento áreas com variação de EVI entre 0,40 e 0,50, o que pode evidenciar áreas utilizadas antropicamente.

A Figura 4 apresenta a Ts na área do $14^{\circ} \mathrm{BIMtz}$ nos anos de 1989, 2006 e 2010. Mais uma vez, observa-se que, ao comparar a temperatura média no interior do objeto de estudo com as temperaturas externas, constata-se uma redução significativa das Ts médias no interior da área militar. A seta, na Figura 4, indica uma área urbanizada na qual foi registrado um aumento da temperatura média de uma faixa de $26,1^{\circ} \mathrm{C}$ a $27,0{ }^{\circ} \mathrm{C}$ para uma faixa de $27,1^{\circ} \mathrm{C}$ a $28,0^{\circ} \mathrm{C}$, gerando um aumento médio de $1,0{ }^{\circ} \mathrm{C}$. Diferentemente, na área da mata do $14^{\circ}$ BIMtz, essa temperatura muda da faixa de $24,1{ }^{\circ} \mathrm{C}$ a $25,0{ }^{\circ} \mathrm{C}$ para $19,1{ }^{\circ} \mathrm{C}$ a $22,0{ }^{\circ} \mathrm{C}$, ou seja, uma redução de aproximadamente $5,0^{\circ} \mathrm{C}$ para um período de 20 anos.

Da mesma forma que as demais áreas, a mata do Complexo Militar do Curado (Figura 5) também indica uma sensível redução da Ts média em toda área de estudo. E, de forma semelhante, as temperaturas nas áreas adjacentes a esse objeto de estudo apresentam valores maiores. Observase, porém, que houve um aumento significativo da temperatura em parte desse objeto (indicado pela seta). Esse aumento de Ts, da ordem de 2,0 ${ }^{\circ} \mathrm{C}$, ocorreu em uma região em que foram construídas, na década de 1990, as instalações do $4^{\circ}$ Batalhão de Polícia de Exército.

\section{CONCLUSÕES}

- Os fragmentos de Mata Atlântica alvos deste estudo apresentam temperaturas mais baixas que suas áreas adjacentes;

- É evidente a melhora da condição climática no interior dos fragmentos analisados. As análises temporais e espaciais declinam uma maior variação relativa entre as Ts registradas no interior e no exterior das matas, evidenciando uma melhora dos serviços ambientais climáticos nas áreas tuteladas ao Exército;

- Essa constatação evidencia o papel desempenhado pelo Exército no controle do desmatamento e na facilitação da regeneração florestal, sendo importante reconhecer tal contribuição para a conservação ambiental no Estado de Pernambuco, incorporando essa instituição no esforço governamental de concepção e implantação da política florestal;

- O método de comparação da Temperatura Superficial por análise do balanço de radiação mostrou-se adequado ao estudo comparativo da evolução ambiental de fragmentos de mata.

\section{STATUS DA SUBMISSÃO}

Recebido: $15 / 02 / 2012$

Aceito: 25/04/2012

Publicado: 30/09/2012

\section{AUTOR(ES) PARA CORRESPONDÊNCIA}

\section{Helder de Barros Guimarães}

Departamento de Engenharia Civil, Universidade Federal de Pernambuco - UFPE, Rua Barão de Itamaracá, 430, Apto 201, CEP 52020-070, Espinheiro, Recife, PE, Brasil e-mail: helder.prof@gmail.com

\section{REFERÊNCIAS}

Accioly LJ, Pacheco A, Costa TCC, Lopes OF, Oliveira MAJ. Relações empíricas entre a estrutura da vegetação e dados do sensor TM/Landsat. Revista Brasileira de Engenharia Agrícola e Ambiental 2002; 6(3): 492-498. http://dx.doi.org/10.1590/S1415-43662002000300019 
Allen RG, Tasumi M, Trezza R. SEBAL - Surface Energy Balance Algorithms for Land. version 1.0. 2002. Advance Training and Users Manual - Idaho Implementation. 97 p. Available from: http://www.dca.ufcg.edu.br/DCA_ download/ISR/UFPE/Final\%20Sebal\%20Manual.pdf.

Almeida TS, Fontana DC, Martorano LG, Bergamasch $H$. Índices de vegetação para a cultura da soja em diferentes condições hídricas e de sistema de manejo. In: Anais do Simpósio Brasileiro de Sensoriamento Remoto; 2005; Goiânia. INPE; 2005. Available from: http://marte.dpi.inpe.br/col/ltid.inpe.br/ sbsr/2004/11.18.17.02/doc/Indices.pdf.

Asner GP. Cloud cover in Landsat observations of the Brazilian Amazon. International Journal of Remote Sensing 2001; 22: 3855-3862. http://dx.doi. org/10.1080/01431160010006926

Baldocchi DD, Meyers TP. On using ecophysiological, micrometeorological and biogeochemical theory to evaluate carbon dioxide, water vapor and trace gas fluxes over vegetation: a perspective. Agricultural and Forest Meteorology 1998; 90(1): 1-25. http://dx.doi. org/10.1016/S0168-1923(97)00072-5

Boegh E, Soegaard H, Thomsen A. Evaluating evapotranspiration rates and surface conditions using Landsat TM to estimate atmospheric resistance and surface resistance. Remote Sensing of Environment 2002; 79(1): 329-343. http://dx.doi. org/10.1016/S0034-4257(01)00283-8

Camargo FF, Pereira G, Moraes EC, Oliveira LGL, Admi M. Análise multitemporal da evolução urbana e sua influência na modificação do campo térmico na Região Metropolitana de São Paulo para os anos de 1985, 1993 e 2003. In: Anais do XIII Simpósio Brasileiro de Sensoriamento Remoto; 2007; Florianópolis. Florianópolis; 2007. p. 5127-5134. Available from: http://marte.dpi.inpe.br/col/dpi.inpe. br/sbsr@80/2006/11.18.00.05/doc/5127-5134.pdf.

Cavalcanti C, organizador. Meio Ambiente, desenvolvimento sustentável e políticas públicas. 4. ed. São Paulo: Cortez; Recife: Fundação Joaquim Nabuco; 2002. 436 p. Available from: http:// www.4shared.com/office/M6JUSSej/meio_ambiente_ desenvolvimento_.html.

Chander G, Markham B. Revised Landsat-5 TM radiometric calibration procedures and postcalibration dynamic ranges. IEEE Transactions on Geoscience and Remote Sensing 2003; 41(11). http://dx.doi.org/10.1109/ TGRS.2003.818464

De Groot RS. Functions of Nature: Evaluation of Nature in environmental planning, management e decision makung. Groningen: WoltersNoordhoff; 1992. 315 p. Available from: http://books. google.com.br/books/about/Functions_of_nature. html?id=rqiZAAAAIAAJ\&redir_esc $=y$.
Giongo PR. Estimativa do balanço de radiação com técnicas de sensoriamento remoto e dados de superfície [dissertação]. Universidade Federal Rural de Pernambuco; 2008. 92 p. Available from: http://200.17.137.108/tde_busca/arquivo. php? $\operatorname{cod}$ Arquivo=339.

Guimarães HB. Serviços Ambientais Climáticos gerados por mata tutelada ao Exército Brasileiro. In: Anais doVI Encontro Internacional das Águas; 2011; Recife. Recife; 2011. Available from: http://www. followscience.com/library/environment-and-agrarian/ ecology/servicos-ambientais-climaticos-gerados-pormata-tutelada-ao-exercito-brasileiro-937.

HueteAR. Adjustingvegetationindicesfor soilinfluences. International Agrophysics 1988; 4(4): 367-376.

Huete AR, Liua HQ, Batchilya K, Van Leeuwen W. A comparison of vegetation indices over a global set of TM images for EOS-MODIS. Remote Sensing of Environment 1997; 59: 440- 451. http://dx.doi. org/10.1016/S0034-4257(96)00112-5

Justice CO, Vermote E, Townshend JRG, Defries R, Roy DP, Hall DK, et al. The Moderate Resolution Imaging Spectroradiometer (MODIS): land remote sensing for global change research. IEEE Transactions on Geoscience and Remote Sensing 1998; 36(4): 1228-1249. http://dx.doi.org/10.1109/36.701075

Leitão MMVBR, Oliveiara GM. Influência da irrigação sobre o albedo. Revista Brasileira de Engenharia Agrícola e Ambiental 2000; 4(2): 214-218.

Lino CF. Texto simples: a Mata Atlântica. RBMA; 2003. [cited 2010 jul. 13]. Available from: http://www.rbma. org.br/anuario/mata_01_sintese.asp.

Machado CCC, Oliveira TH, Galvíncio JD, Silva JS. Utilização do EVI e Albedo da superfície para análise das mudanças ambientais em parte do município de Corumbá, MS e Ladário, MS.In: Anais do II Simpósio de Geotecnologias no Pantanal; 2009; Corumbá. Embrapa Informática Agropecuária/INPE; 2009. p.460-469. Available from: http://www.geopantanal2009.cnptia. embrapa.br/cd/pdf/p130.pdf.

Markhanm BL, Barker LL. Thematic mapper bandpass solar exoatmospherical irradiances. International Journal of Remote Sensing 1987; 8(3): 517-523. http:// dx.doi.org/10.1080/01431168708948658

Oliveira TH, Galvíncio JD. Caracterização ambiental da bacia hidrográfica do Rio Moxotó - PE usando sensoriamento remoto termal. RBGF-Revista Brasileira de Geografia Física Recife-PE 2008; 1(2): 30-49. Available from: http://www.ufpe.br/rbgfe/index.php/revista/ article/view/43/37.

Oliveira TH, Machado CCC, Silva JS, Galvíncio JD, Pimentel RMM, Silva BB. Índice de Umidade (NDWI) e Análise Espaço-Temporal do Albedo da Superfície da Bacia Hidrográfica do Rio Moxotó-PE. Revista 
Brasileira de Geografia Física 2010; 03:55-69. Available from: http://www.ufpe.br/rbgfe/index.php/revista/ article/view/130/106.

Oliveira TH, Silva JS, Machado CCC, Rodrigues GTA, Galvíncio JD, Pimentel RMM. Avaliação espaço-temporal do Índice de área foliar e impacto das atividades antrópicas na Reserva Ecológica Estadual Mata São João da Várzea, Recife PE. In: Anais do XV Simpósio Brasileiro de Sensoriamento Remoto - SBSR; 2011; Curitiba. São José dos Campos: INPE; 2011. v. 1, p. 2105-2112. Available from: http:// www.dsr.inpe.br/sbsr2011/files/p0525.pdf.

Oke TR. Boundary layer climates. 2nd ed. New York; London: Methuen; 1987. 435 p. Available from: http:// books.google.com.br/books/about/Boundary_Layer_ Climates.html?id=1j6rUfnnpS8C\&redir_esc $=y$.

Paiva CM. Estimativa do Balanço de Energia e da Temperatura da Superfície via satélite NOAA-AVHRR [tese]. 208 f. Rio de Janeiro: Universidade Federal do Rio de Janeiro; 2005. Available from: http://www. dca.ufcg.edu.br/DCA_download/ISR/UFPE/Tese_ Doutorado_Celia.pdf

Silva BB, Lopes GM, Azevedo PV. Determinação do albedo de áreas irrigadas utilizando imagens Landsat 5 - TM. Revista Brasileira de Agrometeorologia 2005a; 13(2): 201-211. Available from: http://www.dca.ufcg.edu.br/DCA download/ISR/UFPE/Artigo\%20002-004\%20-\%20 Determina\%E7\%E3o\%20Albedo.pdf.
Silva BB, Lopes GM, Azevedo PV. Balanço de radiação em áreas irrigadas utilizando imagens Landsat 5-TM. Revista Brasileira de Meteorologia 2005b; 20(2): 243252. Available from: http://www.dca.ufcg.edu.br/DCA download/ISR/UFPE/56-2004_BBarbosa-aceito.pdf.

Silva BB, Cândido MV. Determinação da evapotranspiração em escala regional através do Sebal e imagens Landsat 5 - TM. In: Anais do XIII Congresso Brasileiro de Meteorologia; 2004; Fortaleza. Fortaleza, 2004.

Tonhasca A. Os serviços ecológicos da Mata Atlântica. Revista Ciência Hoje 2004; 35(205): 64-67.

Urbanetz C. Estudos florísticos da floresta ombrófila densa atlântica da Fazenda Folha Larga, Cananéia, SP [dissertação]. Campinas: Universidade Estadual de Campinas; 2005. 106 p. Available from: http://www. bibliotecadigital.unicamp.br/document/? code=vtls000 $353544 \& f d=y$.

Viana V, Pinheiro LAFV. Leandro. Conservação da biodiversidade em fragmentos florestais. Serie Tecnica / Instituto de Pesquisas e Estudos Florestais 1988; 12(32): 25-42.

Xia Z, Rui S, Bing Z, Qingxi T. Land cover classification of the North China Plain using MODIS EVI time series. ISPRS Journal of Photogrammetry \& Remote Sensing 2008; 63: 476-484. http://dx.doi.org/10.1016/j. isprsjprs.2008.02.005 